\title{
Threshold based Routing Protocol for WSN with Sleep/Awake Scheduling
}

\author{
Kavita Sharma \\ Research Scholar, \\ Department of Electronics \& Communication Engg. \\ Panipat Institute of Engineering \& Technology \\ Samalkha, Panipat, India
}

\author{
Parveen Singla \\ Assistant Professor \\ Department of Electronics \& Communication Engg. \\ Panipat Institute of Engineering \& Technology \\ Samalkha, Panipat, India
}

\begin{abstract}
Hierarchical routing architecture partitions the whole network in to a group of cluster and only cluster head is responsible for forwarding the data to base station. In this work, we have considered the concept of heterogeneity as in Stable Election Protocol (SEP). In heterogeneous environment, a percentage of the population of sensor nodes is equipped with additional energy resources - this is a source of heterogeneity which may result from the initial setting or as the operation of the network evolves. SEP does not consider the residual energy of a node while electing cluster head to increase reliability as well as there is no concept of sleep nodes to enhance network lifetime. In this paper, we have proposed a routing protocol which will consider the residual energy of a node before making cluster head as well as we have introduced the concept of sleep nodes, so that it will be reliable and energy efficient and results will be compared with existing routing protocol of same category such as SEP.
\end{abstract}

\section{Keywords}

Heterogeneous wireless sensor network, SEP, energy efficient, reliable

\section{INTRODUCTION}

With a sensor network, a user should be able to assign some sensor nodes to monitor specific events, and know when interested events happen in the interested field [1]. Thus, the sensor network builds a bridge between the real world and computation world. Each node typically consists of the five components: sensor unit, Analog Digital Convertor (ADC), Central Processing Unit (CPU), power unit, and communication unit $[1,2]$. The sensor unit is responsible for collecting information as the $\mathrm{ADC}$ requests, and returning the analog data it sensed. ADC is a translator that tells the CPU what the sensor unit has sensed, and also informs the sensor unit what to do. Communication unit is tasked to receive command or query from, and transmit the data from CPU to the outside world. CPU is the most complex unit that interprets the command or query to ADC, monitors and controls power unit if necessary, processes received data, computes the next hop to the sink etc. [3]. Many Routing protocols are existent in literature of wireless sensor network [4].

\section{ROUTING TECHNIQUE IN WSN}

Depending on how the sender of a message gains a route to the receiver, routing protocols can be classified into three categories. Flat routing architecture, hierarchical routing architecture, and location based architecture are one of those.

\subsection{Flat Routing Architecture}

The first category of routing protocols is the multi-hop flat routing protocols. In flat routing, each node typically plays the same role and sensor nodes collaborate to perform the sensing task. Due to the large number of such nodes, it is not feasible to assign a global identifier to each node. This consideration has led to data-centric routing, where the base stations (BS) sends queries to certain regions and waits for data from the sensors located in the selected regions. Since data is being requested through queries, attribute-based naming is necessary to specify the properties of data [5].

\subsection{Hierarchical Routing Architecture}

Hierarchical or cluster-based routing methods, originally proposed in wire line networks are the well-known methods with special advantages related to scalability and efficient communication. As such, the concept of hierarchical routing is also utilized to perform energy efficient routing in WSNs. The main idea in this class of protocols is that every sensor node within a WSN is grouped along with some other of its neighboring nodes so that to constitute a specific cluster. Data collected by the sensor nodes belonging to a cluster are not directly transmitted to the base station. Instead, a node of the cluster, called cluster head, collects these data and forwards them to the base station after possibly having performed appropriate data aggregation [6-8]. In this way, the number of transmitted messages to the base station is reduced and considerable power conservation is achieved. Hierarchical routing can be further of two types as non-centralized and centralized hierarchical routing. In non-centralized type each node self-configures for the cluster head whereas in centralized type of hierarchical routing base station takes this responsibility.

\subsection{Location Based Routing Architecture}

In this kind of routing, sensor nodes are addressed by means of their locations. The distance between neighboring nodes can be estimated on the basis of incoming signal strengths. Relative coordinates of neighboring nodes can be obtained by exchanging such information between neighbors [9-10].

\section{PROPOSED ALGORITHM}

The foundation of proposed protocol lies in the realization that the base station is a high energy node with a continuous energy supply. The proposed protocol uses the base station to control the coordinated sensing task performed by the sensor nodes. The following assumption are to be considered.

- A fixed base station is located in the middle of the region.

- The nodes are equipped with power control capabilities to vary their transmitted power. 
- Each node senses the environment with the fixed rate and always has data to send to the base station.

- All sensor nodes are immobile.The steps of the algorithm are as follows:

1. The radio channel is supposed to be symmetrical. Thus, the energy required to transmit a message from a source node to a destination node is the same as the energy required to transmit the same message from the destination node back to the source node for a given SNR (Signal to Noise Ratio). Moreover, it is assumed that the communication environment is contention and error free. Hence, there is no need for retransmission.

2. A percentage of the population of sensor nodes is equipped with more energy resources than the rest of the nodes. Let $\mathrm{m}$ be the fraction of the total number of nodes $\mathrm{n}$, which are equipped with $\alpha$ times more energy than the others. We refer to these powerful nodes as advanced nodes, and the rest $(1-\mathrm{m}) \times \mathrm{n}$ as a normal nodes. We assume that all nodes are distributed randomly over the sensor field.

3. Suppose that Eo is the initial energy of each normal sensor. The energy of each advanced node is then Eo* $(1+\alpha)$. The total (initial) energy of the new heterogeneous setting is equal to:

$\mathrm{n} * \mathrm{Eo}^{*}(1+\alpha * \mathrm{~m})$

So, the total energy of the system is increased by a factor of $1+\alpha * \mathrm{~m}$.

(i) each normal node becomes a cluster head once every 1 popt $\cdot(1+\alpha \cdot \mathrm{m})$ rounds;

(ii) each advanced node becomes a cluster head exactly $1+\alpha$ times every 1 popt $\cdot(1+\alpha \cdot \mathrm{m})$ rounds;

and (iii) the average number of cluster heads per round per epoch is equal to $\mathrm{n} \times \mathrm{popt}$

Where epoch is the current round and popt is probability to become cluster head.

4. Cluster Head Election for normal nodes is based on following equation:

$$
T\left(s_{n r m}\right)=\left\{\begin{array}{lr}
\frac{P_{n r m}}{1-P_{n r m} \cdot\left(r \cdot \bmod \frac{1}{P_{n r m}}\right)} & \text { if } s_{n r m} \in G^{\prime} \\
0 & \text { otherwise }
\end{array}\right.
$$

where $r$ is the current round, $G^{\prime}$ is the set of normal nodes that have not become cluster heads within the last $\mathbf{1} / \mathbf{P}_{\mathbf{n r m}}$ rounds, and $\mathbf{T}\left(\mathbf{s}_{\mathbf{n r m}}\right)$ is the threshold applied to a population of $\mathrm{n} \cdot(1$ - m) (normal) nodes. This guarantees that each normal node will become a cluster head exactly once every 1/popt. $(1+\alpha \cdot m)$ rounds per epoch, and that the average number of cluster heads that are normal nodes per round per epoch is equal to $n \cdot(1-m) \times$ pnrm.

5. Cluster Head Election for advanced nodes is based on following equation:

$$
T\left(s_{a d v}\right)=\left\{\begin{array}{lr}
\frac{P_{a d v}}{1-P_{a d v}\left(r \cdot \bmod \frac{1}{P_{a d v}}\right)} & \text { if } S_{a d v} \in G^{\prime \prime} \\
0 & \text { otherwise }
\end{array}\right.
$$

where $\mathrm{G}^{\prime \prime}$ is the set of advanced nodes that have not become cluster heads within the last 1 padv rounds of the epoch, and $\mathrm{T}(\mathrm{sadv})$ is the threshold applied to a population of $\mathrm{n} \mathrm{x} \mathrm{m}$ (advanced) nodes. This guarantees that each advanced node will become a cluster head exactly once every $(1 /$ popt $) \mathrm{x}((1+\alpha \cdot \mathrm{m}) /(1+\alpha))$ rounds.

6. Based on above equations and conditions, nodes sends the data to their respective cluster heads and energy consumption will be calculated as

$$
\mathbf{E}_{\text {node }}=\mathbf{k} .\left(\mathbf{E}_{\mathbf{T x} .}(\mathbf{l}, \mathbf{d})+\mathbf{E}_{\mathrm{amp}}\right)
$$

7. Cluster Head will aggregate the data and send it to the base station and energy consumption will be calculated for each node and cluster heads.

$\mathbf{E}_{\text {cluster }}=\mathbf{k} \cdot\left(\mathbf{E}_{\mathrm{TX} .}(\mathbf{l}, \mathbf{d})+\mathbf{E}_{\mathrm{Rx} .}(\mathbf{l})+\mathbf{E}_{\mathrm{DA}}+\mathbf{E}_{\mathrm{amp}}\right.$

8. In round 2, the nodes will become cluster heads according to probability condition i.e. according to minimum distance from base station and threshold energy.

9. After selection of cluster heads, Nodes sends the data to their respective cluster heads, that will be selected according to the minimum distance of a particular node from cluster heads and energy consumption will be calculated.

10. Cluster head will aggregate the data and send it to the base station and energy consumption will be calculated.

11. Ten nodes will also go in sleep mode to enhance the network lifetime if their energy is less than $1 \mathrm{~nJ}$. If the numbers increase then ten, then the nodes will come in active mode and send the data to nearby cluster head.

12. This process will be repeated until the whole network gets down or number of rounds finished. Performance will be evaluated according to parameters like network lifetime, energy dissipation and data packets sent etc.

\section{SIMULATION AND RESULTS}

The simulation is performed using MATLAB. Table 1 shows input parameters that are used for performing simulation.

Table 1 Network Parameters

\begin{tabular}{|c|c|}
\hline Network field area & $200 \times 200 \mathrm{~m}$ \\
\hline $\mathrm{N}$ (Number of nodes) & 100 \\
\hline Initial energy & $1 \mathrm{Joule}$ \\
\hline $\begin{array}{c}\text { Eelec. (Energy dissipation for } \\
\text { Transmitting Energy }\left(\mathrm{E}_{\mathrm{Tx}} \text { \& Receiving }\right. \\
\left.\text { Energy, }\left(\mathrm{E}_{\mathrm{Rx}}\right)\right)\end{array}$ & $50 \mathrm{~nJ} / \mathrm{bit}$ \\
\hline Energy $(\varepsilon)$ for free space & $10 \mathrm{pJ} / \mathrm{bit} / \mathrm{m}^{2}$ \\
\hline Energy $\left(\varepsilon_{\mathrm{mp}}\right.$ for Multipath fading & $0.0013 \mathrm{pJ} / \mathrm{bit} / \mathrm{m}^{4}$ \\
\hline EDA (Energy Aggregation Data) & $5 \mathrm{~nJ} / \mathrm{bit} / \mathrm{signal}$ \\
\hline Data packet size & $4000 \mathrm{bits}$ \\
\hline
\end{tabular}




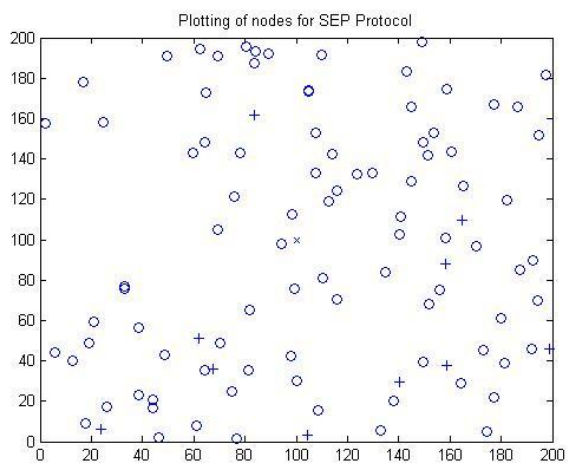

Fig 1. Plotting of nodes for SEP protocol

Refer figure 1, 100 nodes that are randomly deployed in an area of $200 \mathrm{mX} 200 \mathrm{~m}$ for SEP. 'o' symbol denoted as normal nodes, '+' symbol denoted as advanced nodes with extra energy and ' $\mathrm{x}$ ' indicates position of base station.

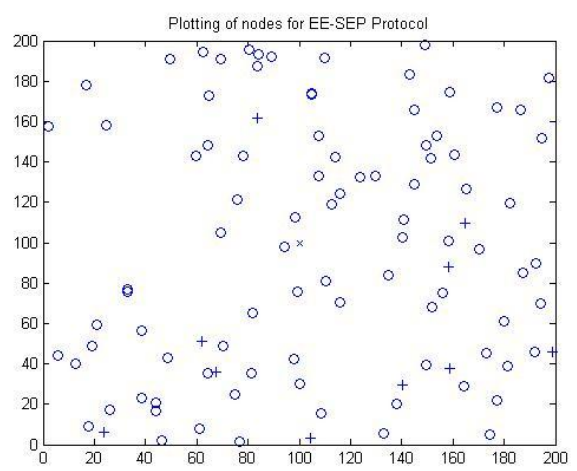

Fig 2. Plotting of nodes for EE-SEP protocol

Refer figure 1, 100 nodes that are randomly deployed in an area of $200 \mathrm{mX} 200 \mathrm{~m}$ for EE-SEP. 'o' symbol denoted as normal nodes, '+' symbol denoted as advanced nodes with extra energy and ' $\mathrm{x}$ ' indicates position of base station.

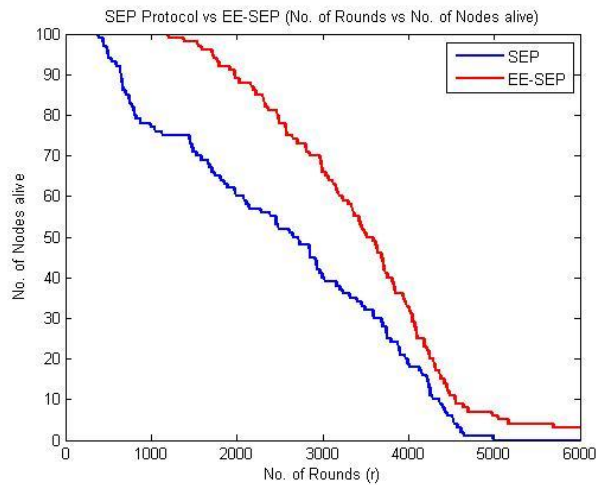

Fig 3. Number of rounds vs. number of nodes alive

Figure 3 shows the comparison of routing protocols Stable Election Protocol (SEP) and Energy Efficient Stable Election Protocol (EE-SEP) in terms of Number of nodes alive. As we can see in figure, when number of rounds increases; the numbers of nodes alive are comparatively less in SEP as compared to EE-SEP, because in proposed technique nodes will go to sleep mode if there energy is less. Figure 3 shows the overall lifetime of the network. Here, we can observe that proposed routing technique performs better as compared to SEP.

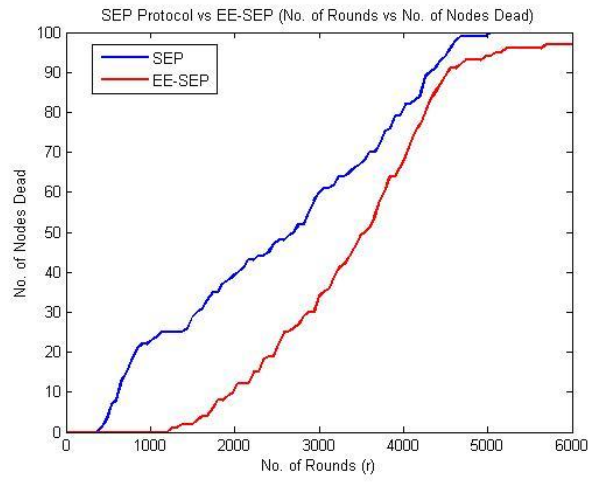

Fig 4. Number of rounds vs. number of nodes dead

Figure 4 shows the comparison of routing protocols Stable Election Protocol (SEP) and Reliable Energy Efficient Stable Election Protocol (EE-SEP) in terms of Number of nodes dead. As we can see in figure, when number of rounds increases; the numbers of nodes dead are comparatively less in EE-SEP as compared to SEP. Figure 4 shows the overall lifetime of the network. Here, we can observe that proposed routing technique performs better as compared to SEP.

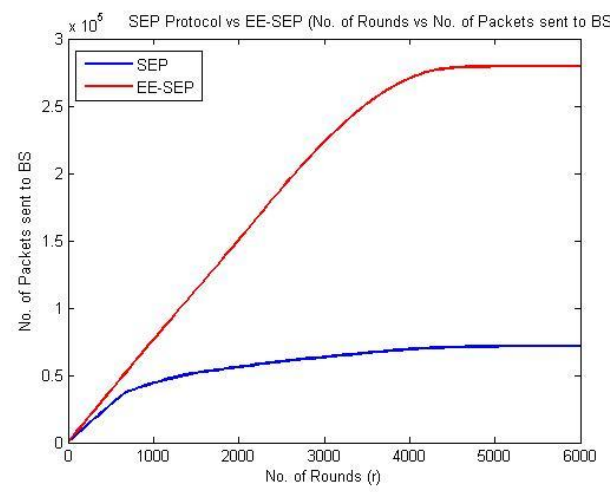

Fig 5. Number of rounds vs. data packets sent to base station

Figure 5 shows how much data will be sent from nodes to base station. From figure 5, we can observed that, in SEP protocol data sent to base station is relatively less as compared to proposed routing technique EE-SEP.

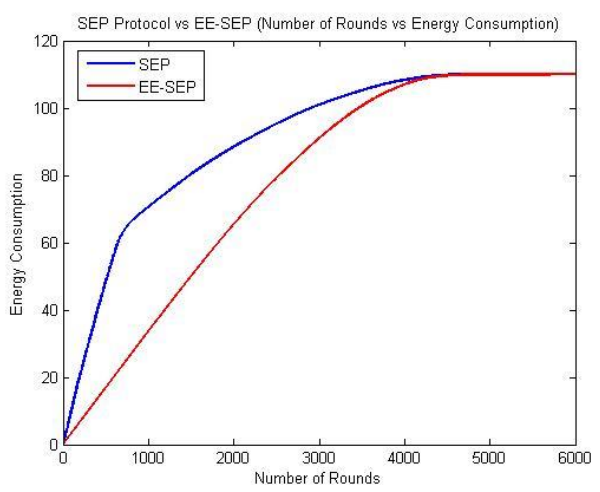

Fig 6. Number of rounds vs. energy consumption in each round 
Figure 6 shows the lifetime of the network. It shows that how energy of $t$ he network consumes round by round and finally whole network goes down. It can be observed from the figure 6 that, EE-SEP consumes less energy and sustain more number of rounds as compare to SEP protocol.

\section{CONCLUSION AND FUTURE WORK}

The proposed routing protocol named Energy EfficientStable Election Protocol (EE-SEP) which is hierarchical routing based with the whole control to the base station or we can say that base assisted. In proposed routing technique, the base station first collects information about the logical structure of the network and residual energy of each node. In EE-SEP, the concept of sleep nodes also enhances the network lifetime. Finally, proposed routing technique is compared with already developed routing protocol Stable Election Protocol (SEP) by the help of MATLAB. A comparison between two is done on the basis of data packet sent and the system lifetime of network.

The proposed technique is energy efficient and reliable, but there is no security and authentication while communicating. So this can be another research area where this can be considered. So in future, security can be applied to proposed routing technique.

\section{REFERENCES}

[1] S. Guo, O.W.W. Yang, "Energy-Aware Multicasting in Wireless Ad hoc Networks: A Survey and Discussion," Computer Communications, Elsevier, April 2007, Vol. 30, Issue 9, pp. 2129-2148.

[2] J. Yick, B. Mukherjee, D. Ghosal, "Wireless Sensor Network Survey," Computer Networks, Elsevier, August 2008, Vol. 52, Issue 12, pp. 2292-2330.

[3] G. Anastasi, M. Conti, M. Francesco, A. Passarella, "Energy Conservation in Wireless Sensor Networks: A survey," Ad Hoc Networks, Elsevier, May, 2009, Vol. 7, Issue 3, pp. 537-568.

[4] R .V. Biradar, V. C.Patil, S. R. Sawant, R. R. Mudholkar, "Classifiacation and Comparison of Routing Protocols in Wireless Sensor Networks," Special Issue on Ubiquitous Computing Security Systems, August 2009, Vol. 4, Issue 2, pp. 704-711.

[5] R. Yadav, S. Varma, N. Malaviya, "A Survey of MAC Protocols for Wireless Sensor Networks," UbiCC Journal, 2009, Vol. 4, Issue 3, pp. 827-833.

[6] S. Ehsan, B. Hamdaoui, "A Survey on Energy-Efficient Routing Techniques with QoS Assurances for Wireless Multimedia Sensor Networks," IEEE Commun. Surveys Tuts., 2011, Vol. 14, Issue 2, pp.265-278.

[7] Nikolaos A. Pantazis, Stefanos A. Nikolidakis and Dimitrios D. Vergados, "Energy-Efficient Routing Protocols in Wireless Sensor Networks: A Survey" IEEE COMMUNICATIONS SURVEYS \& TUTORIALS, VOL. 15, NO. 2, SECOND QUARTER 2013.

[8] Brahim Elbhiri et. Al. "Developed Distributed EnergyEfficient Clustering (DDEEC) for heterogeneous wireless sensor networks", IEEE 2010.

[9] Rashmi Ranjan Sahoo, Moutushi Singh, Biswa Mohan Sahoo, "A Light Weight Trust Based Secure and Energy Efficient Clustering in Wireless Sensor Network: Honey Bee Mating Intelligence Approach", International Conference on Computational Intelligence: Modeling Techniques and Applications (CIMTA), 2013.

[10] Minh Tuan Nguyen and Nazanin Rahnavard , "ClusterBased Energy-Efficient Data Collection in Wireless Sensor Networks utilizing Compressive Sensing", IEEE Military Communications Conference, 2013. 